\title{
Hadron thermodynamics from imaginary chemical potentials
}

\author{
Attila Pásztor ${ }^{1, \star}$, Paolo Alba ${ }^{2}$, Rene Bellwied ${ }^{3}$, Szabolcs Borsányi ${ }^{1}$, Zoltán Fodor ${ }^{1,4,5}$, Jana \\ N. Günther ${ }^{6}$, Sándor Katz ${ }^{5,7}$, Claudia Ratti ${ }^{3}$, Valentina Mantovani Sarti ${ }^{8}$, Jacquelyn Noronha- \\ Hostler $^{9}$, Paolo Parotto ${ }^{3}$, Israel Portillo Vazquez ${ }^{3}$, Volodymyr Vovchenko ${ }^{2,10,11}$, and Horst \\ Stoecker ${ }^{2,10,12}$ \\ ${ }^{1}$ Department of Physics, Wuppertal University, Gaussstr. 20, D-42119, Wuppertal, Germany \\ ${ }^{2}$ Frankfurt Institute for Advanced Studies, Goethe Universität, D-60438 Frankfurt am Main, Germany \\ ${ }^{3}$ Department of Physics, University of Houston, Houston, TX 77204, USA \\ ${ }^{4}$ Jülich Supercomputing Centre, Forschungszentrum Jülich, D-52425 Jülich, Germany \\ ${ }^{5}$ Inst. for Theoretical Physics, Eötvös University, Pázmány P. sétány 1/A, H-1117 Budapest, Hungary \\ ${ }^{6}$ Department of Physics, University of Regensburg, Universitätsstraße 31, D-93053 Regensburg, Germany \\ ${ }^{7}$ MTA-ELTE "Lendület" Lattice Gauge Theory Research Group, Budapest, Hungary \\ ${ }^{8}$ Department of Physics, Technische Universität München, D-85748 Garching, Germany \\ ${ }^{9}$ Department of Physics and Astronomy, Rutgers University, Piscataway, NJ 08854, USA \\ ${ }^{10}$ Institut für Theoretische Physik, Goethe Universität Frankfurt, D-60438 Frankfurt am Main, Germany \\ ${ }^{11}$ Department of Physics, Taras Shevchenko National University of Kiev, 03022 Kiev, Ukraine \\ ${ }^{12}$ GSI Helmholtzzentrum für Schwerionenforschung GmbH, D-64291 Darmstadt, Germany
}

\begin{abstract}
We use 4stout improved staggered lattice data at imaginary chemical potentials to calculate fugacity expansion coefficients in finite temperature QCD. We discuss the phenomenological interpretation of our results within the hadron resonance gas (HRG) model, and the hints they give us about the hadron spectrum. We also discuss features of the higher order coefficients that are not captured by the HRG. This conference contribution is based on our recent papers $[1,2]$.,
\end{abstract}

\section{Introduction}

The vast majority of lattice results for QCD thermodynamics can be described, in the hadronic phase, by a non-interacting gas of hadrons and resonances which includes the measured hadronic spectrum up to a certain mass cut-off. This is known as the Hadron Resonance Gas (HRG) model [3, 4]. The main ingredient of such a model being the hadron spectrum. It has been proposed recently to use the precise lattice QCD results on specific observables, and their possible discrepancy with the HRG model predictions, to infer the existence of higher mass states [5]. We will start this conference contribution by describing how we went further along this direction in our recent paper [1], where we separate thermodynamic contributions from different strageness and baryon number sectors, and pinpoint which chanels have a significant number of states missing.

It is also known, that near the cross-over temperature, the ideal HRG description no longer describes the thermodynamics correctly. It was pointed out recently however, that the behavior of lattice

\footnotetext{
${ }^{\star}$ Speaker
} 
observables in the crossover region, particularly of correlations and fluctuations of conserved charges, are very sensitive to the modeling of baryonic interactions [6, 7]. In many cases including interaction between hadrons changes even the qualitative features of some observables.

This is of great interest, since HRG type model calculations of conserved charge fluctuations are often used to extract freeze-out parameters of heavy ion collisions [8]. Since the freeze-out temperature is close to the cross-over temperature such a sensitivity means that the extracted freeze-out parameters from such a model will depend on the modeling of the hadronic interactions. My second topic in this conference contribution will be about using lattice calculations to constrain models with repulsive interactions between baryons. This is based on our recent paper [2].

For both topics, hadron spectrum and hadronic repulsion, our discussion will be based on the very simple fact that fugacity expansion parameters at a finite real chemical potential become Fourier coefficients at imaginary chemical potential.

\section{Lattice Action}

We use the 4stout improved staggered action, introduced in [9], for both calculations. We carry out simulations at several different values of purely imaginary chemical potentials $[10,11]$. For the study of the hadron spectrum and the strangeness sectors, we use configurations both at $\mu_{B}=\mu_{S}=0$ and $\operatorname{Im} \mu_{S}>0$. In this case we use $N_{t}=10,12,16$ lattices for continuum extrapolation. For studying the repulsive hadronic interactions, we use lattices with $\operatorname{Im} \mu_{B}>0$. For this project we did not carry out a continuum extrapolation, we only use $N_{t}=12$ lattices.

\section{Strange hadrons}

\subsection{Ideal HRG}

In the ideal HRG approach, the pressure is given by:

$$
P_{t o t}\left(T, \mu_{k}\right)=\sum_{k}(-1)^{B_{k}+1} \frac{d_{k} T}{(2 \pi)^{3}} \int d^{3} \vec{p} \ln \left(1+(-1)^{B_{k}+1} \exp \left[-\frac{\left(\sqrt{\vec{p}^{2}+m_{k}^{2}}-\mu_{k}\right)}{T}\right]\right)
$$

where the sum runs over all the hadrons and resonances included in the model. Here the single particle chemical potential is defined with respect to the global conserved charges (baryonic $B$, electric $Q$ and strangeness $S$ ) as $\mu_{k}=B_{k} \mu_{B}+Q_{k} \mu_{Q}+S_{k} \mu_{S}$. For this section of the paper, we will work in the confines of an ideal HRG model, and will try to implement every thermodynamic result in terms this model.

The HRG model provides a good description of several thermodynamic quantities below the phase transition temperature. However, discrepancies are starting to appear when more differential observables are being calculated, such as the fluctuations of conserved charges, defined as:

$$
\chi_{l m n}^{B Q S}=\left(\frac{\partial^{l+m+n} P\left(T, \mu_{B}, \mu_{Q}, \mu_{S}\right) / T^{4}}{\partial\left(\mu_{B} / T\right)^{l} \partial\left(\mu_{Q} / T\right)^{m} \partial\left(\mu_{S} / T\right)^{n}}\right)_{\mu=0} .
$$

For some observables, including new hadron states, predicted by the quark model helps in making the the discrepancy disappear. An important example of such an observable is shown in Figure 1. However, for other observables the agreement between HRG model and lattice gets worse when the QM states are included. For example the QM result overestimates both $\chi_{4}^{S} / \chi_{2}^{S}$ and $\chi_{11}^{u s}$. What does this mean? $\chi_{4}^{S} / \chi_{2}^{S}$ is proportional to the average strangeness squared in the system: the fact that the 


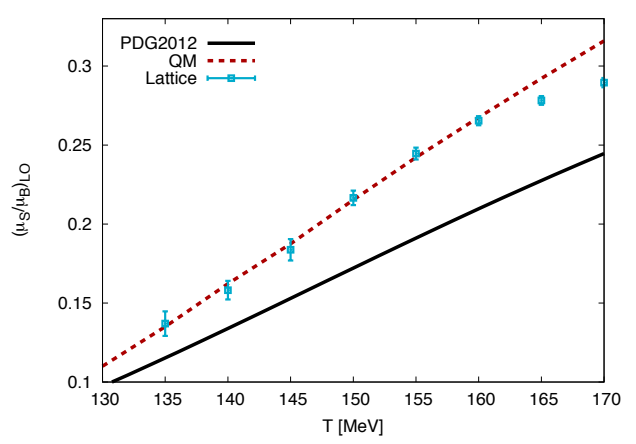

Figure 1. (Color online). Assuming strangeness neutrality, the strangeness chemical potential is given in leading order by $\left(\frac{\mu_{S}}{\mu_{B}}\right)_{L O}=-\frac{\chi_{11}^{B S}}{\chi_{2}^{S}}-\frac{\chi_{11}^{Q S}}{\chi_{2}^{S}} \frac{\mu_{Q}}{\mu_{B}}$. Here we see this combination of conserveced charges from the lattice and from the ideal HRG model. The HRG results are shown for different hadronic spectra, namely by using the PDG2012 (black solid line) and the QM (dashed red line).

QM overestimates it means that it contains either too many multi-strange states or not enough $|S|=1$ states. As for $\chi_{11}^{u s}$, there the contribution is positive for baryons and negative for mesons, so the QM overestimating it means that either it contains too many strange baryons or not enough strange mesons. The bottom line is that the interpretation of these comparisons with the HRG is ambiguous. In order to more effectively settle this issue alternative observables are needed.
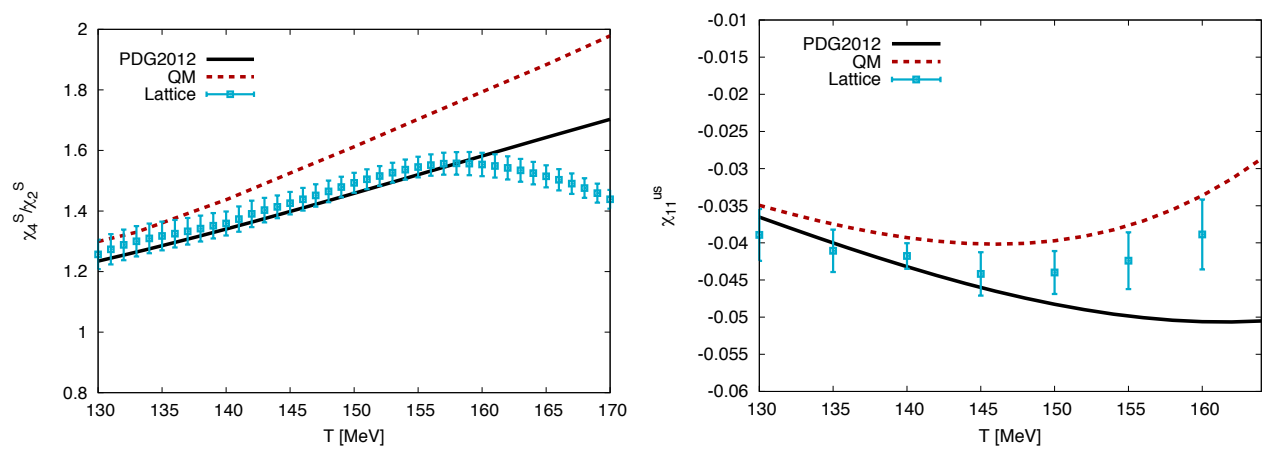

Figure 2. (Color online). Left: Ratio $\chi_{4}^{S} / \chi_{2}^{S}$ as a function of the temperature. HRG model calculations based on the PDG2012 (black solid line) and the QM (red dashed line) spectra are shown in comparison to the lattice results from Ref. [12]. Right: comparison of up-strange correlator $\chi_{11}^{u s}$ simulated on the lattice [9] and calculated in the HRG model using the PDG2012 (solid black line) and the QM (dashed red line) spectra. 


\subsection{Imaginary chemical potential and strangeness sectors}

In order to solve this ambiguity, we separate the pressure of QCD as a function of the temperature into contributions coming from hadrons grouped according to their quantum numbers:

$$
\begin{array}{r}
P\left(\hat{\mu}_{B}, \hat{\mu}_{S}\right)=P_{B=0, S=0}+P_{|B|=1,|S|=0} \cosh \left(\hat{\mu}_{B}\right)+P_{B=0,|S|=1} \cosh \left(\hat{\mu}_{S}\right)+P_{|B|=1,|S|=1} \cosh \left(\hat{\mu}_{B}-\hat{\mu}_{S}\right) \\
+P_{|B|=1,|S|=2} \cosh \left(\hat{\mu}_{B}-2 \hat{\mu}_{S}\right)+P_{|B|=1,|S|=3} \cosh \left(\hat{\mu}_{B}-3 \hat{\mu}_{S}\right)
\end{array}
$$

where $\hat{\mu}_{i}=\mu_{i} / T$. This particular truncation of the fugacity expansion of the pressure is motivated by our assumption that in the confined phase, a HRG with some hadron spectra describes the thermodynamics of the QCD system.

Assuming this ansatz for the pressure, the partial pressures $P_{|B|=i,|S|=j}$ can be expressed as linear combinations of the susceptibilities $\chi_{i j}^{B S}$. For example $P_{B=0,|S|=1}=\chi_{2}^{S}-\chi_{22}^{B S}$. This means that in principle we can determine the strangeness sectors just by using the usual fluctuation observables. This is not the approach we pursue here. Instead, we simulate with an imaginary strangeness chemical potential $\mu_{S}=i \mu_{I}$. In this case by simple differentiation one gets:

$$
\begin{aligned}
\operatorname{Im} \chi_{1}^{B} & =-P_{|B|=1,|S|=1 \mid} \sin \left(\mu_{I}\right)-P_{|B|=1,|S|=2} \sin \left(2 \mu_{I}\right)-P_{|B|=1,|S|=3} \sin \left(3 \mu_{I}\right), \\
\chi_{2}^{B} & =P_{|B|=1, S=0}+P_{|B|=1,|S|=1} \cos \left(\mu_{I}\right)+P_{|B|=1,|S|=2} \cos \left(2 \mu_{I}\right)+P_{|B|=1, S \mid=3} \cos \left(3 \mu_{I}\right), \\
\operatorname{Im} \chi_{1}^{S} & =\left(P_{B=0,|S|=1}+P_{1|1|}\right) \sin \left(\mu_{I}\right)+2 P_{|B|=1,|S|=2} \sin \left(2 \mu_{I}\right)+3 P_{|B|=1,|S|=3} \sin \left(3 \mu_{I}\right),
\end{aligned}
$$

and similarly for $\chi_{2}^{S}, \chi_{3}^{S}$ and $\chi_{4}^{S}$. The advantage of the imaginary chemical potential approach is that, even though it is based on the exact same assumption as the direct evaluation of linear combinations, it reduces the errors considerably, since the lower derivatives already contain the information on the higher strangeness sectors.
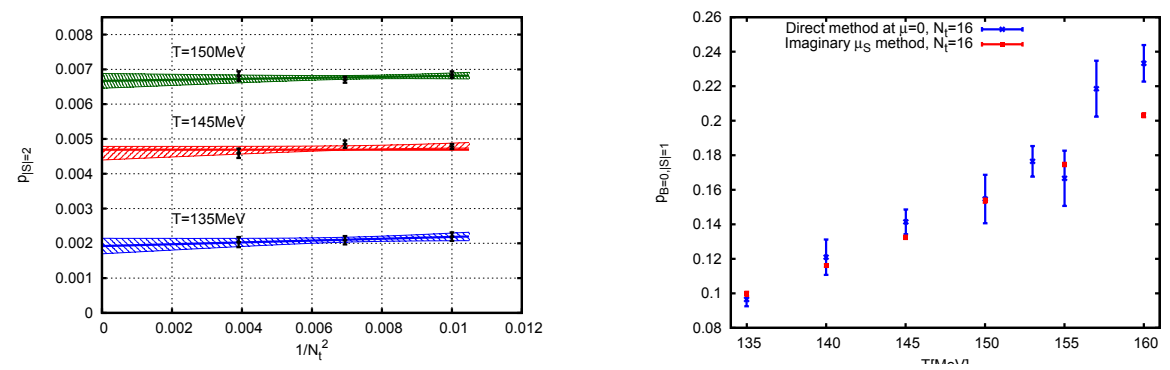

Figure 3. Left: Examples of continuum limit extrapolation from our $N_{t}=10,12,16$ lattices. Right: Comparison of our method to Taylor expansion from $\mu=0$ data for $P_{01}$. The statistics would explain only a factor of 2 difference in the errorbars, but the improvement is much more drastic than that.

To obtain the Fourier cofficients $\left(P_{B=0,|S|=1}+P_{|B|=1,|S|=1}\right), P_{|B|=1,|S|=2}$ and $P_{|B|=1,|S|=3}$ we perform a correlated fit with the previous ansatz for the observables $\chi_{1}^{S}, \chi_{2}^{S}, \chi_{3}^{S}$ and $\chi_{4}^{S}$ at every temperature. To obtain $P_{|B|=1, S=0}$ we fit $\chi_{2}^{B}$, to obtain $P_{|B|=1,|S|=1}$ we fit $\chi_{1}^{B}$. To get $P_{|B|=0,|S|=1}$ we just take the difference $\left(P_{B=0,|S|=1}+P_{|B|=1,|S|=1 \mid}\right)-P_{|B|=1,|S|=1}$. For statistical errors, we use the jacknife method. For the continuum limit we use $N_{t}=10,12$ and 16 lattices. For systematic errors on the continuum extrapolation we use two different scale settings: $w_{0}$ and $f_{\pi}$. More details on the scale setting can be found in [9]. We also include two different interpolating functions for temperature dependence of the $P_{B S}$ : a third order polynomial $a+b T+c T^{2}+d T^{3}$ and a rational function $\left(a+b T+c T^{2}\right) /(1+d T)$. Both of 
these describe the data well. We also do the continuum limit with and without tree level improvement factors for the pressure. The continuum limit is done with a linear $a+b / N_{t}^{2}$ and a $1 /\left(a+b / N_{t}^{2}\right)$ ansatz. These 8 different results are then weighted with the AKAIKE information criterion using the histogram method [13]. Examples of linear continuum limit extrapolations are included in Fig. 3(left). For the $B=0,|S|=1$ sector, which includes a large contribution from kaons, the continuum extrapolation could not be carried out using these lattices. Therefore we only include results for the mesonic $|S|=1$ partial pressure $P_{B=0,|S|=1}$ and the total $S=1$ partial pressure $\left(P_{B=0,|S|=1}+P_{|B|=1,|S|=1}\right)$ for finite lattices in this work. The results of our lattice study are summarized in Figure 4. Our analysis clearly shows that the well-established states listed in the most updated version of the PDG are not enough to correctly reproduce the lattice results. The minimal choice which reproduces most of the data is the PDG2016 with the inclusion of one-star states which are not well-established. In some cases, such as the strange mesons and $|S|=3$ baryons, there is room to add even more states. These results will potentially affect all phenomenological applications of the HRG model, including thermal fits and other calculations relevant to heavy-ion physics.
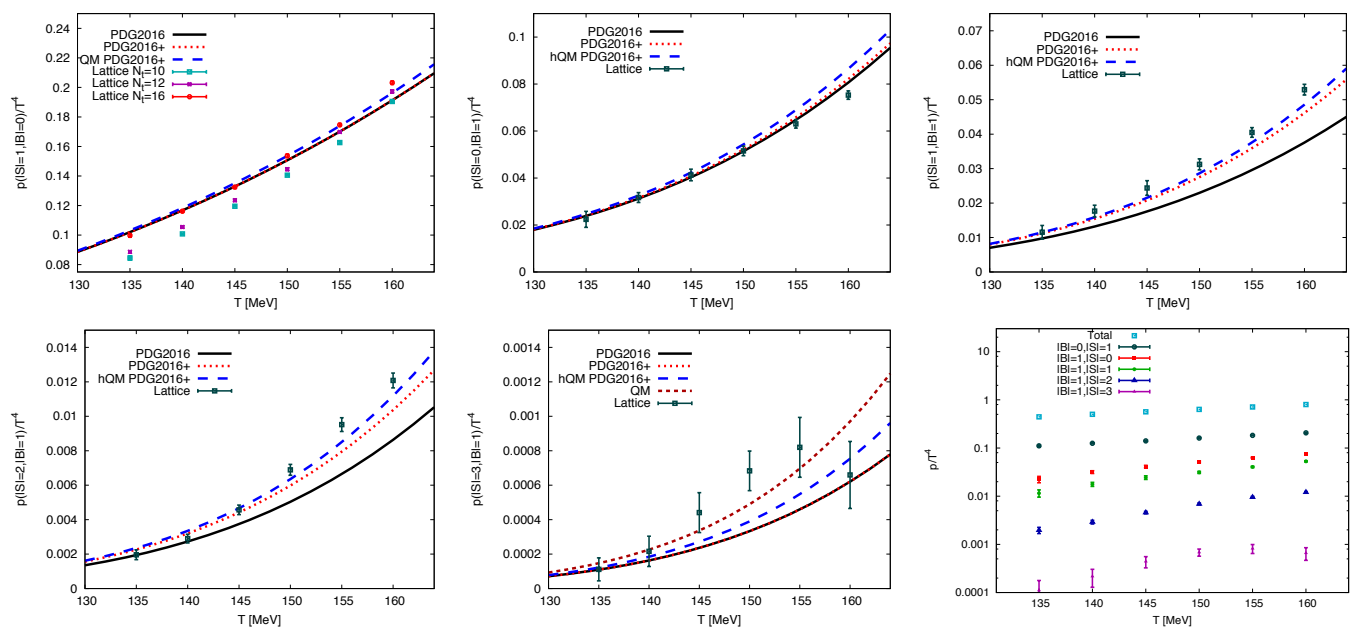

Figure 4. (Color online). Comparison between the lattice results for the partial pressures and the HRG model predictions. Upper panels: Contribution to the total pressure from strange mesons (left), non-strange baryons (center), $|S|=1$ baryons (right). Lower panels: $|S|=2$ baryons (left), $|S|=3$ baryons (center), relative magnitude of the single contributions with respect to the total (right). In the first five panels, the points are the lattice results, while the curves are PDG2016 (solid black), PDG2016+ (including one star states, red dotted), PDG2016+ and additional states from the hQM (blue, dashed) [14, 15]. In the lower center panel, the dark red short-dashed curve corresponds to the PDG2016+ with inclusion of the $\Omega$ resonances predicted by the QM $[16,17]$.

\section{Repulsive baryonic interactions}

For this section we only consider the $\mu_{S}=\mu_{Q}=0$ case, and keep only $\mu_{B}$ to be nonzero. The pressure can be written as:

$$
\frac{p\left(T, \mu_{B}\right)}{T^{4}}=\sum_{k=0}^{\infty} P_{|B|=k}(T) \cosh \left(k \mu_{B} / T\right),
$$


While the baryon number for $\mu_{B}=i \tilde{\mu}_{B}$ is:

$$
\chi_{1}^{B}\left(T, i \tilde{\mu}_{B}\right)=i \sum_{k=1}^{\infty} b_{k}(T) \sin \left(k \tilde{\mu}_{B} / T\right),
$$

where $b_{k}=k P_{|B|=k}$. The coefficients can easily be calculated by a Fourier transformation. The main difference between this and the previous section (the strangeness sectors) is that in this case we do not want to commit to a particular truncation of the series expansion (5), instead, we want the Fourier analysis tell us, when a component appears. In the ideal HRG model with no light nuclei included $b_{1}>0$ and $b_{2}=b_{3}=b_{3}=\cdots=0$. The inclusion of nuclei would induce nonzero $b_{2}, b_{3}, \ldots$, but always with a positive sign. In contrast, at very large temperatures, where we have free quarks, we expect:

$$
\frac{p^{\mathrm{SB}}}{T^{4}}=\frac{8 \pi^{2}}{45}+\sum_{f=u, d, s}\left[\frac{7 \pi^{2}}{60}+\frac{1}{2}\left(\frac{\mu_{f}}{T}\right)^{2}+\frac{1}{4 \pi^{2}}\left(\frac{\mu_{f}}{T}\right)^{4}\right] .
$$

Since we only consider the case $\mu_{S}=\mu_{Q}=0$, one has $\mu_{f}=\mu_{B} / 3$. The net baryon susceptibility at imaginary $\mu_{B}$ reads

$$
\chi_{1}^{B}\left(T, i \tilde{\mu}_{B}\right)=\left.\frac{\partial\left(p / T^{4}\right)}{\partial\left(\mu_{B} / T\right)}\right|_{\mu_{B}=i \tilde{\mu}_{B}}=\frac{i}{3}\left[\frac{\tilde{\mu}_{B}}{T}-\frac{1}{9 \pi^{2}}\left(\frac{\tilde{\mu}_{B}}{T}\right)^{3}\right] .
$$

The coefficients $b_{k}^{\mathrm{SB}}$ are calculated by a Fourier integral to be:

$$
b_{k}^{\mathrm{SB}}=\frac{(-1)^{k+1}}{k} \frac{4\left[3+4(\pi k)^{2}\right]}{27(\pi k)^{2}} .
$$

Since this sign alternating behavior is strikingly different from the HRG expectation of $b_{2}=b_{3}=$ $b_{4}=\cdots=0$, one might interpret the appearance of an alternating sign structure as a signal for deconfinement.

Such a conclusion might be hasty however. As we point out in [2], hadronic models can also produce such a sign structure. The simple model we use to illustrate this is the HRG with an excluded volume repulsive interaction between baryons and baryons, anti-baryon and anti-baryons, and no interactions between other hadron pairs. We call this model EV-HRG. The model has one free parameter, an average excluded volume parameter $b$. Model calculations of the $b_{k}$ can be found in [2]. The first Fourier coefficient $b_{1}$ does not depend on this $b$ parameter, the higher coefficients do, but once $b$ is adjusted such that $b_{2}$ is reproduced, near $T_{c}$, it appears that also $b_{3}$ and $b_{4}$ are described well by the model. This can be seen in Figure 5 .

The success of the EV-HRG model in describing these four Fourier coefficients does not automatically mean that such a model describes all other QCD observables, for instance the correlations and fluctuations involving the electric charge and strangeness, in the same temperature range. These observables are sensitive to the baryon-baryon interactions as well. At the same time, they are also sensitive to interactions involving mesons, as these carry both electric charge and strangeness, and to the strangeness-dependent baryonic interactions. These extensions are beyond the scope of the present work. The main point we would like to make is that these fugacity expansion parameters are quite sensitive to the modeling of hadronic interactions, and therefore can be quite useful when trying to adjust hadronic modelling.

One might wonder how the fugacity expansion parameters relate to the more conventional observables, the fluctuations of conserved charges. This is illustrated in Figure 6. When the effects of baryon-baryon interactions are small, the third and higher order coefficients can be neglected, and $\chi_{2}^{B}-\chi_{4}^{B}$ is just proportional to $b_{2}$. This fact was exploited in [18]. 

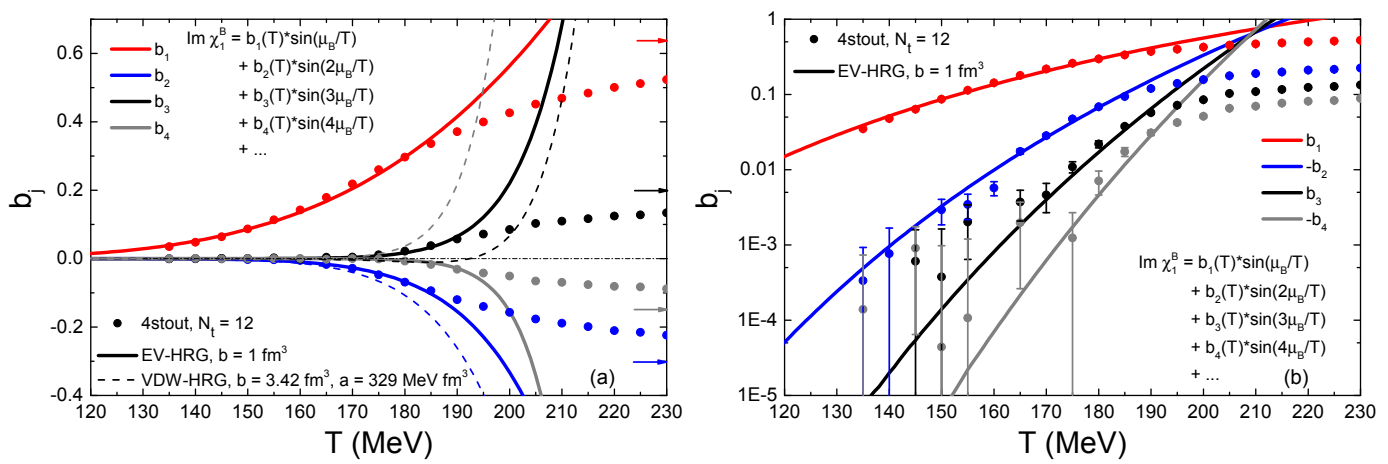

Figure 5. The temperature dependence of the first four Fourier coefficients $b_{k}$, calculated on the lattice at $N_{t}=12$ (symbols), and within the EV-HRG model with baryonic eigenvolume parameter $b=1 \mathrm{fm}^{3}$ (solid lines). This dependence is shown on (a) the linear and (b) the logarithmic scales. The dashed lines in (a) show the calculations within the VDW-HRG model, with van der Waals parameters $a$ and $b$ fixed by the properties of the nuclear ground state [6]. The arrows in (a) correspond to the Stefan-Boltzmann limit (9).

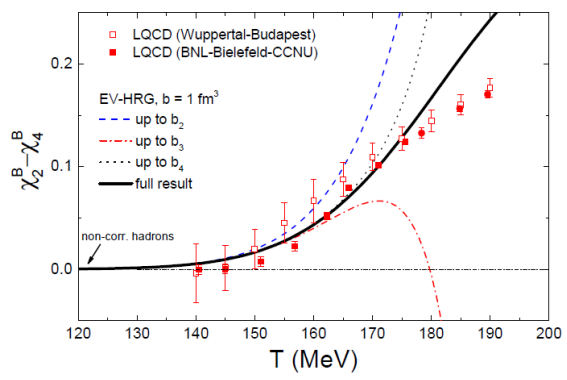

Figure 6. The temperature dependence of $\chi_{2}^{B}-\chi_{4}^{B}$, calculated within the EV-HRG model with baryonic eigenvolume parameter $b=1 \mathrm{fm}^{3}$ (solid line). Other lines depict the EV-HRG model calculations using truncations of the fugacity expansion, at the second (dashed blue line), third (dash-dotted red line), and fourth (dotted black line) orders. Lattice QCD data from Refs. [9] and [19] are depicted, respectively, by open and full symbols.

\section{Summary}

Lattice simulations at imaginary chemical potentials allow one to separate thermodynamic contributions from sectors with different conserved charges. In this conference contribution we briefly explained two phenomelogical applications of this fact. First, we with in an ideal HRG model, and looked for missing strange resonances by separating sectors in baryon number and strangeness, with simulations in the confined phase and with $\operatorname{Im} \mu_{s}>0$. We clearly saw, that the well-established states listed in PDG 2016 are not enough to correctly reproduce the lattice results. Next, we looked at extending the HRG model with repulsive baryonic interactions. Here the higher order fugacity expansions in the baryon number proved useful, as we demonstrated these to be sensitive to repulsion between baryons. We saw that repulsion between hadrons can reproduce the sign structure seen in the higher order fugacity expansion coefficients, and that the lattice data constrain the excluded volume parameter to be around $1 \mathrm{fm}^{3}$. 


\section{Acknowledgements}

This work was supported by HIC for FAIR within the LOEWE program of the State of Hesse. V.V. acknowledges the support from HGS-HIRe for FAIR. H.St. acknowledges the support through the Judah M. Eisenberg Laureatus Chair at Goethe University. This project was funded by the DFG grant SFB/TR55. This material is based upon work supported by the National Science Foundation through grant number NSF PHY-1654219 and by the U.S. Department of Energy, Office of Science, Office of Nuclear Physics, within the framework of the Beam Energy Scan Theory (BEST) Topical Collaboration. The work of R. Bellwied is supported through DOE grant DEFG02-07ER41521. An award of computer time was provided by the INCITE program. This research used resources of the Argonne Leadership Computing Facility, which is a DOE Office of Science User Facility supported under Contract DE-AC02-06CH11357. The authors acknowledge the Gauss Centre for Supercomputing (GCS) for providing computing time for a GCS Large-Scale Project on the GCS share of the supercomputer JUQUEEN [20] at Jülich Supercomputing Centre (JSC), and at HazelHen supercomputer at HLRS, Stuttgart. The authors acknowledge the use of the Maxwell Cluster and support from the Center of Advanced Computing and Data Systems at the University of Houston.

\section{References}

[1] P. Alba et al., Phys. Rev. D96, 034517 (2017), 1702 . 01113

[2] V. Vovchenko, A. Pasztor, Z. Fodor, S.D. Katz, H. Stoecker (2017), 1708.02852

[3] R. Dashen, S.K. Ma, H.J. Bernstein, Phys. Rev. 187, 345 (1969)

[4] R. Venugopalan, M. Prakash, Nucl. Phys. A546, 718 (1992)

[5] A. Bazavov, H.T. Ding, P. Hegde, O. Kaczmarek, F. Karsch et al., Phys.Rev.Lett. 113, 072001 (2014), 1404.6511

[6] V. Vovchenko, M.I. Gorenstein, H. Stoecker, Phys. Rev. Lett. 118, 182301 (2017), 1609. 03975

[7] V. Vovchenko, Phys. Rev. C96, 015206 (2017), 1701.06524

[8] P. Alba, W. Alberico, R. Bellwied, M. Bluhm, V. Mantovani Sarti, M. Nahrgang, C. Ratti, Phys. Lett. B738, 305 (2014), 1403. 4903

[9] R. Bellwied, S. Borsanyi, Z. Fodor, S.D. Katz, A. Pasztor, C. Ratti, K.K. Szabo, Phys. Rev. D92, 114505 (2015), 1507.04627

[10] P. de Forcrand, O. Philipsen, Nucl. Phys. B642, 290 (2002), hep-lat/0205016

[11] M. D’Elia, M.P. Lombardo, Phys. Rev. D67, 014505 (2003), hep-lat/0209146

[12] R. Bellwied, S. Borsanyi, Z. Fodor, S.D. Katz, C. Ratti, Phys.Rev.Lett. 111, 202302 (2013), 1305.6297

[13] S. Durr, Z. Fodor, J. Frison, C. Hoelbling, R. Hoffmann, S.D. Katz, S. Krieg, T. Kurth, L. Lellouch, T. Lippert et al., Science 322, 1224 (2008), 0906. 3599

[14] M. Ferraris, M.M. Giannini, M. Pizzo, E. Santopinto, L. Tiator, Phys. Lett. B364, 231 (1995)

[15] J. Ferretti, R. Bijker, G. Galatà, H. García-Tecocoatzi, E. Santopinto, Phys. Rev. D94, 074040 (2016), 1506.07469

[16] S. Capstick, N. Isgur, Phys.Rev. D34, 2809 (1986)

[17] D. Ebert, R. Faustov, V. Galkin, Phys.Rev. D79, 114029 (2009), 0903.5183

[18] P. Huovinen, P. Petreczky (2017), 1708.00879

[19] A. Bazavov et al., Phys. Rev. D95, 054504 (2017), 1701.04325

[20] JUQUEEN:, Ibm blue gene/q supercomputer system at the julich supercomputing centre, tech. rep.1 al (julich supercomputing centre http://dx.doi.org/10.17815/jlsrf-1-18 2015), http://dx . doi.org/10.17815/jlsrf-1-18). 